\title{
Role of the direct processes in low-energy deuteron interactions
}

\author{
Marilena Avrigeanu ${ }^{\mathrm{a}}$, Vlad Avrigeanu ${ }^{\mathrm{b}}$, and Cristian Costache ${ }^{\mathrm{c}}$ \\ Horia Hulubei National Institute for Physics and Nuclear Engineering, PO Box MG-6, 077125 Bucharest-Magurele, Romania
}

\begin{abstract}
An extended analysis of the key role of direct interactions, i.e., breakup, stripping and pick-up processes, has been carried out for deuteron-induced reactions. Particular comments concern the deuteron breakup which is the dominant mechanism involved in surrogate reactions on heavy nuclei, around the Coulomb barrier.
\end{abstract}

\section{Introduction}

At present increased deuteron-data needs follow the demands of on-going strategic research programs (ITER, IFMIF, SPIRAL2-NFS [1]) that involve deuteron beams, while the corresponding experimental and evaluated data are less extensive and accurate than for neutrons. There are currently many efforts to improve the description of deuteron reactions (e.g., [2]) also due to the use of $(d, p \gamma)$, and $(d, p f)$ surrogate reactions for neutron capture $(n, \gamma)$ and induced fission $(n, f)$ cross sections investigations respectively, of interest for breeder reactors.

The consistent account of the direct interactions (DI) role in deuteron interaction process [3-5], i.e., the breakup (BU) and direct reactions (DR) (stripping and pick-up), forms the object of this work for nuclei from ${ }^{27} \mathrm{Al}$ to ${ }^{238} \mathrm{U}$. While the above-mentioned references include a detailed description of the involved model assumptions and consistent parameters sets that were either established or validated using various independent data, we briefly mention here only the updated main points of these analyzes.

\section{Deuteron breakup}

Concerning the physical picture of the deuteron breakup in the Coulomb and nuclear fields of the target nucleus, two distinct processes are considered in this respect, namely the elastic breakup (EB) in which the target nucleus remains in its ground state and none of the deuteron constituents interact with it, and the inelastic breakup or breakup fusion (BF), where one of these deuteron constituents interacts nonelastically with the target nucleus. The reactions induced by the BF nucleons lead to different compound nuclei than following the incoming deuterons, the partition of the BF cross section among various residual nuclei being triggered by the energy spectra of the BF nucleons and the excitation functions of the pre-equilibrium (PE) and compoundnucleus $(\mathrm{CN})$ reactions induced by these nucleons on the target nuclei [3-5].

\footnotetext{
a e-mail: marilena.avrigeanu@nipne.ro

b e-mail: vlad.avrigeanu@nipne.ro

c e-mail: cristian.costache@nipne.ro
}

The BU cross sections have been obtained from an empirical parametrization [6] of both the total $(\mathrm{EB}+\mathrm{BF})$ breakup proton-emission and $\mathrm{EB}$ fractions, $f_{B U}^{p}=$ $\sigma_{B U}^{p} / \sigma_{R}$ and $f_{E B}=\sigma_{E B} / \sigma_{R}$, respectively, where $\sigma_{R}$ is the deuteron total-reaction cross section. At the same time the inelastic-breakup fraction is given by the difference $f_{B F}^{p}=f_{B U}^{p}-f_{E B}$. The comparison of the parametrization values and experimental $f_{B U}^{p}$ and $f_{E B}$ fractions [9], for target nuclei from ${ }^{27} \mathrm{Al}$ to ${ }^{232} \mathrm{Th}$, is shown in Fig. 1 for deuteron energies up to $30 \mathrm{MeV}$ (left) corresponding to the experimental data basis [9] for the $f_{E B}$ parametrization, as well as in the extended energy range up to $80 \mathrm{MeV}$ (right) of the experimental data basis for the $f_{B U}^{p}$ parametrization.

A first comment concerns the apparent decrease of $f_{E B}$ at incident energies beyond the range within which it was established. Since this trend has been opposite to that of $f_{B U}^{p}$ fraction, in the absence of any available deuteron $\mathrm{EB}$ data at $E_{d}>30 \mathrm{MeV}$, the correctness of the extrapolation in the case of the EB parametrization has been checked by the comparison of its predictions and results of the Continuum-Discretized Coupled-Channels (CDCC) method [7]. The good agreement between the CDCC results, EB parametrization predictions [6], and data systematics [9] has increased the confidence on both CDCC approach and empirical-parametrization basic grounds at energies of the available data (Figs. 2 and 3 of Ref. [7]).

On the other hand, the behavior of CDCC results over $30 \mathrm{MeV}$ has been in agreement only with the $f_{B U}^{p}$ parametrization. Thus it has resulted that the extension of the $f_{E B}$ empirical parametrization beyond the energies at which the corresponding data were available should be considered with caution. Therefore, in order to avoid laborious CDCC calculations for each target/energy of interest, we have adopted a normalized EB fraction for the energies beyond its maximum by taking into account the CDCC results and the behavior of the $f_{B U}^{p}$ fraction. Hence, we have chosen to keep unchanged the ratio of the EB and $\mathrm{BU}$ fractions for the incident energies above the maximum of EB fraction $f_{E B}\left(E_{d, \max }\right)$ by means of the relation

$$
f_{E B}^{n o r m}\left(E_{d}\right)=f_{B U}^{p}\left(E_{d}\right) \frac{f_{E B}\left(E_{d, \max }\right)}{f_{B U}^{p}\left(E_{d, \max }\right)}, \quad E_{d}>E_{d, \max }
$$

(C) The Authors, published by EDP Sciences. This is an Open Access article distributed under the terms of the Creative Commons Attribution License 4.0 (http://creativecommons.org/licenses/by/4.0/). 


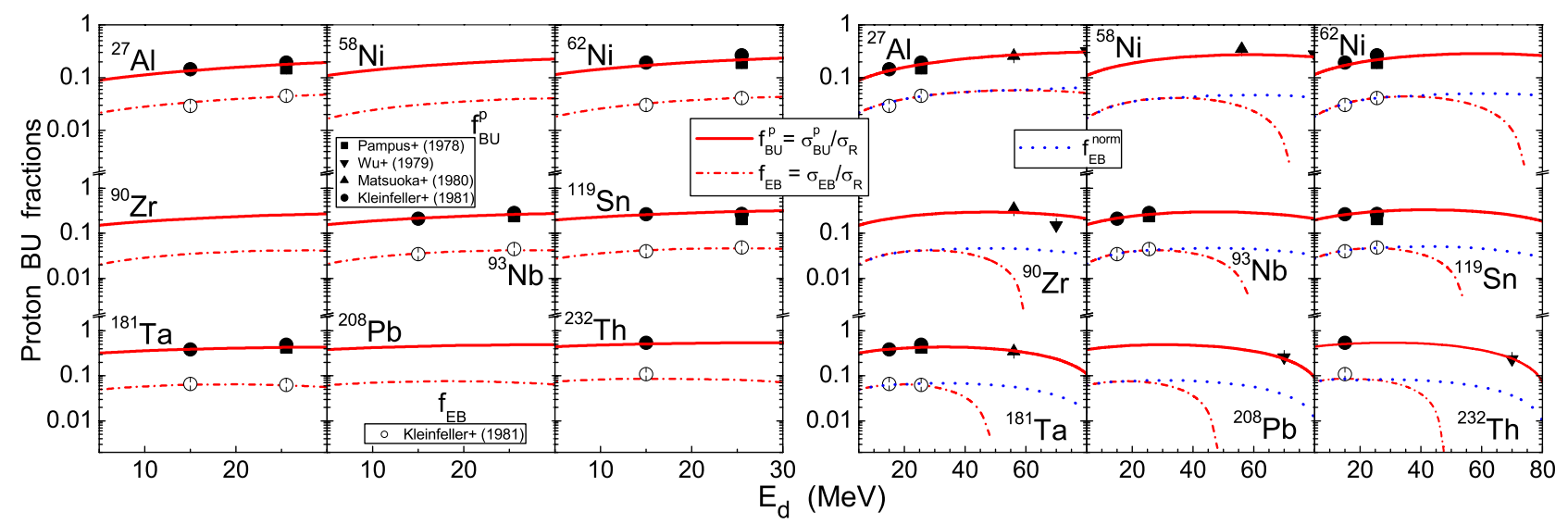

Figure 1. Comparison of experimental [9] breakup proton-emission (solid symbols) and elastic-breakup (open circles) fractions, and the corresponding parametrizations [6] (solid and dash-dotted curves, respectively), for deuterons on nuclei from ${ }^{27} \mathrm{Al}$ to ${ }^{232} \mathrm{Th}$ at incident energies up to $30 \mathrm{MeV}$ (left) and $80 \mathrm{MeV}$ (right), as well as the normalized EB fractions (dotted) for the higher energies (right).

where the energy $E_{d, \max }$ corresponds to the abovementioned maximum. Thus, the normalized EB fraction follows the behavior of total breakup proton-emission fraction as it is also shown in Fig. 1, in agreement with the CDCC calculation results [7]. This $f_{E B}$ normalization is of particular interest, at deuteron energies above $\sim 50 \mathrm{MeV}$ and especially for heavier target nuclei where $E_{d, \max }$ is lower, for both inelastic- and total-breakup fractions [6]:

$$
f_{B F}^{p / n}=f_{B U}^{p / n}-f_{E B}^{n o r m}, \quad f_{B U}=2 f_{B U}^{p / n}-f_{E B}^{n o r m},
$$

assuming equal proton and neutron BU cross sections.

On the other hand, the experimental systematics of $f_{B U}^{p}$ [9] includes only one data for heavy nuclei $(A>200)$ at incident energies around the Coulomb barrier, which are of great interest for surrogate reaction study, namely for ${ }^{232} \mathrm{Th}$ at $E_{d}=15 \mathrm{MeV}$. The present parametrization describes well this data as well as the one at a medium energy of $70 \mathrm{MeV}$ (Fig. 1). However, within the energy range between $\sim 25$ and $41 \mathrm{MeV}$, i.e. around the $E_{d, \text { max }}=$ $32 \mathrm{MeV}$ for this target nucleus, the corresponding total BU fraction exceeds unity (e.g., $f_{B U}=1.0215$ at $E_{d, \text { max }}$ ). Consequently, we have adopted for $A>200$ the additional constraint that the $f_{B U}$ fraction should be less than $90 \%$. It has been included as well as the normalization by the use of Eq. (1) in the code TALYS-1.8 [13], corresponding to the value $\mathbf{2}$ of the breakupmodel option for the BU model calculations.

\section{Direct reactions}

Usually neglected or very poorly taken into account, the direct reactions play an important role in the deuteron interactions at energies around the Coulomb barrier. Their contribution is important for the first-chance emitted particle cross section [3-6].

The $(d, p)$ and $(d, n)$ stripping, and $(d, t)$ and $(d, \alpha)$ pick-up cross sections were calculated using the distorted-wave Born approximation (DWBA) method, within FRESCO code [11], with details given elsewhere [3-6]. Their correctness has been validated by the suitable description of the experimental specific data, e.g. angular distributions and double-differential cross sections. Therefore, the description of the measured [12] populations of low-lying levels in ${ }^{239} \mathrm{U}$ and ${ }^{237} \mathrm{U}$ through
${ }^{238} \mathrm{U}(\mathrm{d}, \mathrm{p}){ }^{239} \mathrm{U}$ and ${ }^{238} \mathrm{U}(\mathrm{d}, \mathrm{t}){ }^{237} \mathrm{U}$ stripping and pick-up reactions, respectively, shown in Fig. 2(a,b), validates the correctness of the corresponding $(d, p)$ and $(d, t)$ total excitation functions in Fig. 2(c, bottom). Sum of the total $(d, p),(d, t)$, and BU cross sections gives a lower limit of the DI contribution to the deuteron interaction with ${ }^{238} \mathrm{U}$ target nucleus.

Finally, the deuteron total-reaction cross section that remains to be available for the $\mathrm{PE}+\mathrm{CN}$ lengthy mechanisms has to be corrected for the incident-flux leakage through DI processes, 1.e. the breakup, stripping and pick-up, by a reduction factor [3-6]:

$$
1-\frac{\sigma_{B U}+\sigma_{(d, p)}+\sigma_{(d, t)}}{\sigma_{R}}=1-\frac{\sigma_{D I}}{\sigma_{R}} .
$$

The reduction factor shown on top of Fig. 2(c) points out the DI dominant role (mainly breakup) in the deuteron interaction with ${ }^{238} \mathrm{U}$ around Coulomb barrier, which is a specific feature in the case of heavy nuclei $[8,10]$.

\section{DI effects on surrogate reactions}

Since last decade the surrogate reaction method [14-16] has been intensively utilized to measure mainly $(n, \gamma)$ and $(n, f)$ cross sections by means of surrogate reactions like $(d, p \gamma)$ and $(d, p f)$. The "desired" $(n, \gamma)$ cross section for a target nucleus $A$ is given in terms of $\mathrm{CN}$ formation cross section $\sigma_{n}^{C N}\left(E_{e x}, J, \pi\right)$ and the branching ratio $G_{\gamma}^{C N}\left(E_{e x}, J, \pi\right)$ toward the desired $\gamma$ outgoing channel:

$$
\sigma_{n, \gamma}\left(E_{n}\right)=\Sigma_{J, \pi} \sigma_{n}^{C N}\left(E_{e x}, J, \pi\right) G_{\gamma}^{C N}\left(E_{e x}, J, \pi\right),
$$

where $J, \pi$ are the spin and parity of the excited state $E_{e x}$.

Usually $\sigma_{n}^{C N}\left(E_{e x}, J, \pi\right)$ is provided by a neutron optical model potential, while theoretical branching ratios $G_{\gamma}^{C N}\left(E_{e x}, J, \pi\right)$ are often quite uncertain [14]. They are extracted in the surrogate method by measuring in $(d, p \gamma)$ surrogate reaction the probability $P_{d, p \gamma}^{\text {exp }}\left(E_{e x}\right)$ for the formation of the same excited-nucleus states, with the same specific $E_{e x}$ and $J, \pi$ values, decaying through the $\gamma$-emission channel. The probability of this surrogate excited-nucleus decay through the $\gamma$ channel is [14]:

$$
P_{d, p \gamma}\left(E_{e x}\right)=\Sigma_{J, \pi} F_{d, p}^{C N}\left(E_{e x}, J, \pi\right) G_{\gamma}^{C N}\left(E_{e x}, J, \pi\right),
$$



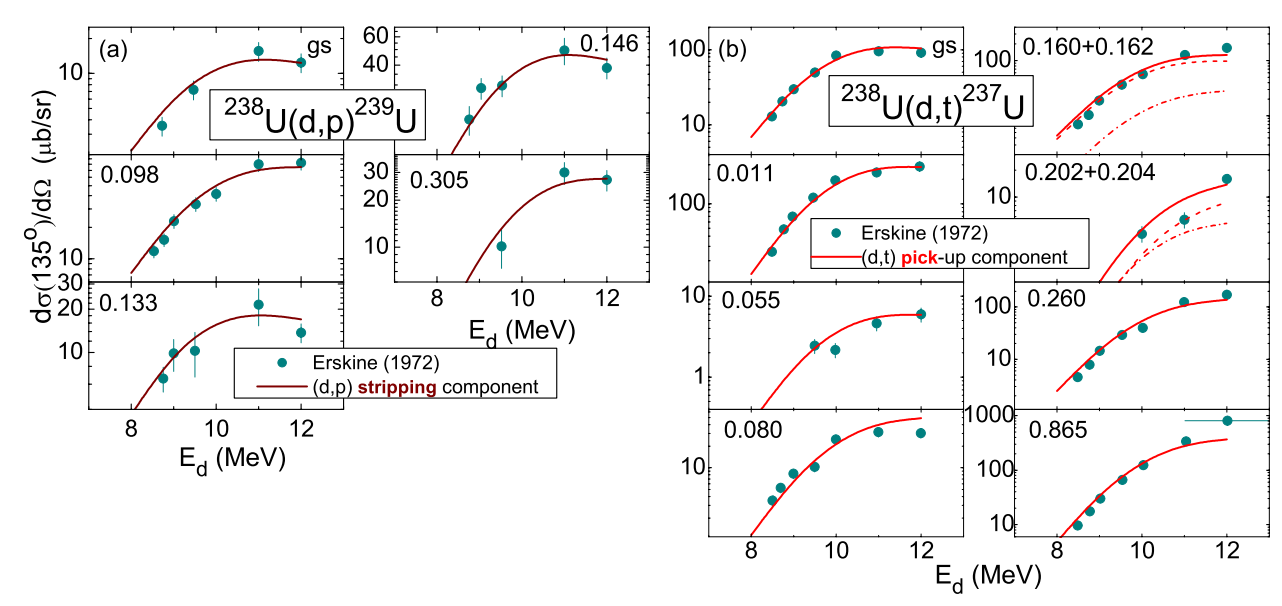

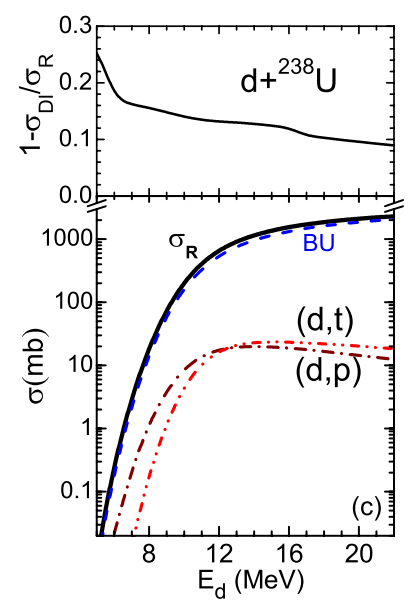

Figure 2. Comparison of calculated (solid curves) and measured [12] excitation functions for the populations at $135^{\circ}$ of low-lying levels in (a) ${ }^{239} \mathrm{U}$ and (b) ${ }^{237} \mathrm{U}$, through $(d, p)$ and $(d, t)$ direct reactions, respectively. (c) Total-reaction (solid curves), breakup (dashed curves), stripping $(d, p)$ (dash-dotted curves), and pick-up $(d, t)$ (dash-dot-dotted curves) cross sections for deuterons on ${ }^{238} \mathrm{U}$ (bottom), and the reduction factor of the deuteron flux going towards statistical processes (bottom).

where $F_{d, p}^{C N}\left(E_{e x}, J, \pi\right)$ is the corresponding probability for the formation of desired excited nucleus in the surrogate reaction. Moreover, $P_{d, p \gamma}\left(E_{e x}\right)$ is obtained experimentally by measuring the total number of the surrogate events given by, e.g., proton spectrum, and the number of coincidences between the surrogate ejectile and the $\gamma$ decay channel, namely, the number of $p-\gamma$ coincidences:

$$
P_{d, p \gamma}^{\text {exp }}\left(E_{e x}\right)=\frac{N_{p, \gamma}^{\text {coincidences }}\left(E_{e x}\right)}{N_{d, p}^{\text {surrogateevents }}\left(E_{e x}\right)} .
$$

However, it is obvious that $P_{d, p \gamma}^{\exp }$ of Eq. (5) does not included the contributions of $C N$ mechanism only. Finally, simplifying the theoretical frame by additional assumptions of (i) similar $J^{\pi}$ distributions in both desired and surrogate reactions, and (ii) no $J^{\pi}$-dependence of the decay probabilities $G_{\gamma}\left(E_{e x}, J, \pi\right)$ (the Weisskopf-Ewing model), the desired neutron capture cross section becomes [14]:

$$
\sigma_{n, \gamma}\left(E_{n}\right)=\sigma_{n}^{C N}\left(E_{n}\right) P_{d, p \gamma}^{\exp }\left(E_{e x}\right) .
$$

The validation test of the deuteron surrogate method by comparing well known $(n, \gamma)$ reaction cross sections with those obtained by analysis of surrogate $(d, p \gamma)$ reaction stressed out large discrepancies $([15,16]$ and Refs. therein) which rise question marks on this method. The reported results at variance from the surrogate ${ }^{238} \mathrm{U}(d, p \gamma){ }^{239} \mathrm{U}$ reaction at the incident energy of $15 \mathrm{MeV}$, and 'desired' ${ }^{238} \mathrm{U}(n, \gamma){ }^{239} \mathrm{U}$ reaction, for incident-neutron energies from 0 to $1.5 \mathrm{MeV}$, by Ducasse et al. [16] are involved hereafter particularly to underline the DI effects.

First, the decay probabilities $P_{d, p \gamma}\left(E_{e x}\right)$ of the residual nucleus ${ }^{239} \mathrm{U}$ were measured by Ducasse et al. at the excitation energies between the corresponding neutronbinding energy $S_{n}=4.806 \mathrm{MeV}$ and $1.5 \mathrm{MeV}$ above it. The protons from $15 \mathrm{MeV}$ deuteron-induced $(d, p \gamma)$ reaction on the ${ }^{238} \mathrm{U}$ target nucleus, corresponding to this excitation energy range, have energies between 12.65 and 11.15 MeV (Fig. 3). These energies are matched also by the $15 \mathrm{MeV}$ deuteron-breakup protons, with the maximum value $E_{p, \text { max }}^{B U}=12.65 \mathrm{MeV}$. Thus, these BU protons may affect the the $P_{d, p \gamma}^{\exp }\left(E_{e x}\right)$ measurement.

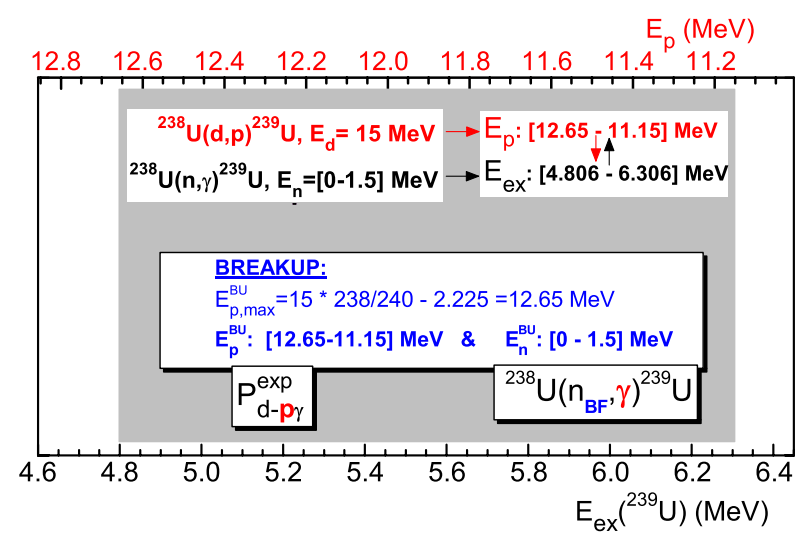

Figure 3. Correlation of residual excitation energies in ${ }^{238} \mathrm{U}(n, \gamma){ }^{239} \mathrm{U}$ desired reaction at $E_{n}=0-1.5 \mathrm{MeV}$ [16] (bottom, in black) and energies of emitted protons in ${ }^{238} \mathrm{U}(d, p \gamma){ }^{239} \mathrm{U}$ surrogate reaction at $E_{d}=15 \mathrm{MeV}$ (top, in red), as well as breakup-nucleon energies for $15 \mathrm{MeV}$ deuterons (insert, in blue) and the perturbed measurements.

Second, the BU protons are correlated with BU neutrons with energies between 0 and $1.5 \mathrm{MeV}$. Moreover, the BF component of these BU neutrons may interact with the ${ }^{238} \mathrm{U}$ target nucleus, populating actually the desired compound nucleus ${ }^{239} \mathrm{U}$ at the same excitation energies of interest. Thus, the $\gamma$-decay of the ${ }^{239} \mathrm{U}^{*}$ populated through ${ }^{238} \mathrm{U}\left(\mathrm{n}_{B F}, \gamma\right)$ reaction may also affect, together with the companion $\mathrm{BF}$ protons, the measured $p-\gamma$ coincidence events and, finally, the $P_{d, p \gamma}^{\exp }\left(E_{e x}\right)$ measurement.

Third, the probability $F_{d, p}^{C N}\left(E_{e x}, J, \pi\right)$ given by Eq. (5) for forming the excited nucleus ${ }^{239} \mathrm{U}$ is decreased due to the incident flux leakage through DI, according to Eq. (3) and Fig. 2(c), as well as the PE processes which may precede the population of the excited nucleus ${ }^{239} \mathrm{U}$ through the $\mathrm{CN}$ reaction: $\mathrm{d}+{ }^{238} \mathrm{U} \rightarrow{ }^{240} \mathrm{~Np}^{*} \rightarrow \mathrm{p}+{ }^{239} \mathrm{U}^{*}$.

Actually, it is a laborious task to select the contribution of $\mathrm{CN}$ mechanism, i.e., the only one considered in the theoretical frame of surrogate reactions, from the measured probabilities $P_{d, p \gamma}^{\exp }\left(E_{e x}\right)$. It involves several corrections concerning the processes left out. In this respect, the simple correction applied in Ref. [16] only 
to the single proton spectrum in Eq. (6) does not take into account neither the strongly reduced $F_{d, p}^{C N}\left(E_{e x}, J, \pi\right)$ probability of forming ${ }^{239} \mathrm{U}^{*}$ nucleus by the deuteron flux leakage through DI and PE processes, nor the contributions of other than $\mathrm{CN}$ mechanism to the population of this nucleus.

Moreover, the assumption concerning the equality of the branching ratios for the deuteron surrogate and the neutron-induced reactions does not hold, due to the population and decay differences between the excited and compound nuclei formed in surrogate and desired reactions, respectively [17]. However, one should be more careful in assuming that the failure of the surrogatemethod validation tests follows the use of the too weak Weisskopf-Ewing approximation $[15,16]$. It is also even the use of the Hauser-Feshbach formalism alone, within deuteron-surrogate reactions analysis, which can not lead to expected good results in the absence of the unitary account of all $\mathrm{BU}+\mathrm{DR}+\mathrm{PE}+\mathrm{CN}$ reaction mechanisms [10].

Finally it is obvious that the hard approximations that led to Eq. (7) are not appropriate for deuteron induced reactions. Therefore, the theoretical frame of deuteron surrogate method should be revised for a consistent account of the reaction mechanisms involved in the complex deuteron interactions.

\section{Conclusions}

The present work discussed a deeper analysis of the key role of DI, particularly of the breakup mechanism, in deuteron-induced reactions. Firstly, a normalization of the parametrized [6] EB fraction for $E_{d}$ values beyond the energy range within it was established, $E_{d} \approx 25-30 \mathrm{MeV}$, has been provided in order to follow the behavior of the total BU proton fraction, in agreement with CDCC results.

Next we analyzed the contributions of breakup, stripping, and pick-up mechanisms to $\mathrm{d}+{ }^{238} \mathrm{U}$ interaction process, at energies around Coulomb barrier, to illustrate the importance of direct interaction processes.

Finally, the validation test of ${ }^{238} \mathrm{U}(\mathrm{d}, \mathrm{p} \gamma)$ surrogate reaction has been discussed. The disregard of DI mechanisms in the theoretical frame of deuteron surrogatereaction method should be considered the main reason for the failure of the $(d, p \gamma)$ validation tests $[15,16]$. However, the surrogate ratio method should be valid in the presence of the deuteron breakup without assuming its specific effects, due to their possible cancellations [17].

This work was partly supported by Fusion for Energy (F4E-GRT168-02) and Unitatea Executiva pentru Finantarea Invatamantului Superior, a Cercetarii, Dezvoltarii si Inovarii (Project No. PN-IIID-PCE-2011-3-0450).

\section{References}

[1] www.iter.org/proj; www.ifmif.org/b/; pro. ganil-spiral2.eu/spiral2/ instrumentation/nfs

[2] J. Lei and A.M. Moro, Phys. Rev. C 92, 044616 (2015); G. Potel, F.M. Nunes, and I.J. Thompson, ibid. 92, 034611 (2015)

[3] P. Bém et al., Phys. Rev. C 79, 044610 (2009); E. Šimečková et al., ibid. 84, 014605 (2011)

[4] M. Avrigeanu et al., Phys. Rev. C 88, 014612 (2013); ibid. 89, 044613 (2014); ibid. 94, 014606 (2016)

[5] M. Avrigeanu et al., Phys. Rev. C 92, 021601(R) (2015); Nucl. Data Sheets 118, 301 (2014)

[6] M. Avrigeanu et al., Fus. Eng. Design 84, 418 (2009)

[7] M. Avrigeanu and A.M. Moro, Phys. Rev. C 82, 037601 (2010)

[8] M. Avrigeanu, V. Avrigeanu, and A.J. Koning, Phys. Rev. C 85, 034603 (2012)

[9] J. Pampus et al., Nucl. Phys. A311, 141 (1978); N. Matsuoka et al., ibid. 345, 1 (1980); J. Kleinfeller et al., ibid. 370, 205 (1981); J.R. Wu et al., Phys. Rev. C 19, 370 (1979); M.G. Mustafa et al., ibid. 35, 2077 (1987)

[10] M. Avrigeanu et al., J. Phys: Conf. Ser. 724, 012003 (2016); CERN-Proceedings-2015-001, pp. 203

[11] I.J. Thompson, Comput. Phys. Rep. 7, 167 (1988); v. FRES 2.9 (2011)

[12] J.R. Erskine, Phys. Rev. C 5, 959 (1972)

[13] A.J. Koning, S. Hilaire, and S. Goriely, v. TALYS1.8, 2016, http: //www.talys.eu

[14] J. Escher et al., Rev. Mod. Phys. 84, 353 (2012)

[15] J.N. Wilson et al., Phys. Rev. C 85, 034607 (2012); B.L. Goldblum et al., ibid. 85, 054616 (2012); J.M. Allmond et al., ibid. 79, 054610 (2009)

[16] Q. Ducasse et al., Phys. Rev. C 94, 024614 (2016)

[17] S. Chiba and O. Iwamoto, Phys. Rev. C 81, 044604 (2010) 